\title{
Sample handling for kinetics and molecular assembly in flow cytometry
}

\author{
Larry A. Sklar, ${ }^{\mathrm{b}}$, Larry C. Seamerb, Frederick Kuckuck ${ }^{b}$ Richard G. Posner, \\ Eric Prossnitz, Bruce Edwards ${ }^{b}$, and John P. Nolan ${ }^{b}$ \\ "National Flow Cytometry Resource, Los Alamos National Laboratory, \\ 'School of Medicine, University of New Mexico, \\ 'Department of Chemistry, Northern Arizona University.
}

\begin{abstract} OSTI.

Flow cytometry discriminates particle associated fluorescence from the fluorescence of the surrounding medium. It permits assemblies of macromolecular complexes on beads or cells to be detected in real-time with precision and specificity. We have investigated two types of robust sample handling systems which provide sub-second resolution and high throughput: 1) mixers which use stepper-motor driven syringes to initiate chemical reactions in msec time frames; and 2) flow injection controllers with valves and automated syringes used in chemical process control. In the former system, we used fast valves to overcome the disparity between mixing $100 \mu \mathrm{ls}$ of sample in $100 \mathrm{msecs}$ and delivering sample to a flow cytometer at $1 \mu \mathrm{l} / \mathrm{sec}$. Particles were detected within $100 \mathrm{msec}$ after mixing, but turbulence was created which lasted for $1 \mathrm{sec}$ after injection of the sample into the flow cytometer. We used optical criteria to discriminate particles which were out of alignment due to the turbulent flow. Complex sample handling protocols involving multiple mixing steps and sample dilution have also been achieved. With the latter system we were able to automate sample handling and delivery with intervals of a few seconds. We used a fluidic approach to defeat turbulence caused by sample introduction. By controlling both sheath and sample with individual syringes, the period of turbulence was reduced to $\sim 200$ msecs. Automated sample handling and sub-second resolution should permit broad analytical and diagnostic applications of flow cytometry.
\end{abstract}

RECEIVED JUL 011998

Keywords: flow cytometry, kinetics, ligand binding, cell activation, fluorescence, screening, sample handling, automation

\section{INTRODUCTION}

There are several complementary goals for handling biological samples for research and industrial applications. In particular, there is a need for assay systems which are robust, sensitive, quantitative, and compatible with high throughput screening for both cells and isolated molecules. In several accompanying articles from this symposium we provide evidence for the utility of flow cytometry as a tool for the investigation of molecular interactions of native molecules on cells and purified molecules on beads.

Flow cytometry is based upon detecting the fluorescence and light scatter signals of particles flowing in a stream past a laser beam. The particles are aligned upon exiting a small nozzle and hydrodynamically focused by coaxial flow of the sample stream within a flowing sheath. The utility of time-base flow cytometry has been recognized for some time (Martin and Schwartzendruber, 1981). Flow cytometry is now routinely used for observing continuously both the binding of soluble molecules to cell surface molecules and cell activation which occurs in response to the binding interaction (Sklar, 1987; Nolan et al., 1998). If cell responses are occurring in time frames of seconds, then one can imagine that the molecular binding events which precede them may include sub-second steps. Over the past decade, there have been numerous designs which provided on-line sample addition of reagents to cell suspensions as they pass through a flow cytometer (Dive et al., 1987; Kachel et al., 1982; Kelly, 1989; Lindberg et al., 1995; Omann et al. 1985). There were two common limitations to the time resolution of these early devices. First, if the flow rate of the sample was $\sim 1 \mu \mathrm{l} / \mathrm{sec}$ between the point that it was being mixed and the point it was being observed in the flow cytometer and the volume between these points was several $\mu l s$, then several seconds could elapse between mixing and observation. The second problem concerns the uncertainty of the efficiency of mixing. Diffusional mixing is slow in large volumes, and even magnetic stir bars requires several seconds for homogeneous mixing of several mis.

Further author information:

L.A.S (correspondence) Email: LSklar@salud.unm.edu. Phone: 505-272-6892; Fax 505-272-6995; J.P.N Email: nolan@telomere.lani.gov 
Our approach has been to adapt rapid mixing technology to flow cytometry (Nolan et al., 1995). This approach enables complete mixing, sample delivery, and initiation of measurement in 300 msecs. The mixing occurs through the turbulent delivery of cells and reagents through a mixing tee. The main obstacle to measurements at earlier times is the turbulence which occurs in the nozzle immediately after the volume between the sample line and the nozzle of the flow cytometer is cleared. The turbulence occurs because the dead volume of a few microliters must be cleared with sample flow at a rate greater than can be accommodated by laminar flow in the nozzle. At flow rates common to flow cytometers, turbulence was detected for $\sim 1$ second after the sample was "injected" into the nozzle. By including in the analysis only those particles which remained aligned in the stream, we were able to obtain times as early as 300 msecs after mixing. The uniformity of mixing was verified by showing that quenching reactions involving fluorescent beads and soluble quenchers were complete by the time the particles were observed.

Recently, coaxial mixing of a sample stream and a reagent stream has allowed time points as early as 100 msec (Scampavia et al., 1995). This approach does not require a turbulence-inducing boost to clear a dead volume rapidly for early time measurements. Rather, sample and reagent flow continuously, and the age of the mixed sample depends upon the point of measurement relative to the point of mixing. Rapid mixing and coaxial mixing are fundamentally different in their approaches and capabilities. While the coaxial approach is free of turbulence, enabling measurements at very early times after mixing, it measures only a single time point in each experiment. The mixing apparatus is physically repositioned prior to the measurement of each subsequent time point. The ability to continuously monitor a single time point allows an unlimited number of cells to be measured at each time point for excellent statistics and this would be an ideal way to analyze and even sort for a sub-population of cells which responds to a stimulus within a defined time window. On the other hand, with stopped flow mixing, once the turbulence has passed, the kinetics of fluorescence change is measured continuously providing the overall time course of a process. Further, it is difficult to conceive of a coaxial mixing geometry which would enable complex mixing protocols. Such complex mixing schemes are extremely valuable for mechanistic studies of cellular and biochemical processes and for high throughput screening. Thus, at this point, the two approaches are somewhat complementary. While coaxial mixing provides superior resolution at early times by avoiding turbulent flow, rapid mixing provides an experimentally flexible system. To further control turbulence at early times following mixing we have adopted the strategy of simultaneously controlling sample and sheath (Seamer et al., submitted). This approach also allows for the programmed delivery of small sample volumes in short time periods.

In this article, we describe the progress we have made in developing or integrating from commercial sources the appropriate tools for sample handling for cytometers. The developments include: 1) recent advances in sample mixing and sample delivery technology which allows kinetic flow cytometric measurements to be made in the sub-second time frame and 2) computerization of sample handling which allows for automation in sample delivery for high throughput screening.

\section{METHODS}

Cell Culture and Reagents. Human neutrophils were handled as described previously (Fay et al., 1991). Human myeloid (U937) cell transfectants expressing the human formyl peptide receptor were cultured in RPMI media at $5 \% \mathrm{CO}_{2}$ in $\mathrm{T}-75$ flasks (Costar). Cells were pelleted by centrifugation, resuspended in buffer ( $30 \mathrm{mM}$ HEPES, $110 \mathrm{mM} \mathrm{NaCl}, 10 \mathrm{mM} \mathrm{KCl}, 1$ $\mathrm{mM} \mathrm{MgCl}, 10 \mathrm{mM}$ glucose, $1 \mathrm{mg} / \mathrm{ml} \mathrm{BSA}, \mathrm{pH} 7.3$ ) at a concentration of $1 \times 10^{7} / \mathrm{ml}$ and stored on ice until use. The fluorescent formyl peptide (N-formyl-met-leu-phe-lys-FITC, Penninsula, Laboratories) was dissolved in DMSO at a concentration of $1 \mathrm{mM}$, and diluted to a working solution of $\sim 1 \mathrm{uM}$. Actual peptide concentrations were determined by absorbance at $490 \mathrm{~nm}$ using an extinction coefficient of $8.1 \times 10^{4} \mathrm{M}^{-1} \mathrm{~cm}^{-1}$ (see Fay et al., 1991). A soluble unlabeled formyl peptide (f-met-leu-phe-phe-gly-gly-lys) and a low affinity labeled peptide (f-met-gly-phe-phe-lys-FITC) were synthesized by Commonwealth Biotechnologies, Inc. (Richmond, Va).

Calibration. The flow cytometer signal was calibrated using standardized fluorescein microspheres (Flow Cytometry Standards Corp, San Juan, PR). Data were analyzed using IDLYK software (NFCR, LANL). Cells were gated using forward and $90^{\circ}$ light scatter, and the mean fluorescence at vs. time was calculated. Mean channel numbers were converted to ligand molecules per cell using the FITC standard bead data and the relative quantum yield $\left(Q_{r}\right)$ of conjugated fluorescein compared to free fluorescein as describer in Fay et al. (1991). 


\section{DISCLAIMER}

This report was prepared as an account of work sponsored by an agency of the United States Government. Neither the United States Government nor any agency thereof, nor any of their employees, makes any warranty, express or implied, or assumes any legal liability or responsibility for the accuracy, completeness, or usefulness of any information, apparatus, product, or process disclosed, or represents that its use would not infringe privately owned rights. Reference herein to any specific commercial product, process, or service by trade name, trademark, manufacturer, or otherwise does not necessarily constitute or imply its endorsement, recommendation, or favoring by the United States Government or any agency thereof. The views and opinions of authors expressed herein do not necessarily state or reflect those of the United States Government or any agency thereof. 


\section{DISCLAIMER}

Portions of this document may be illegible electronic image products. Images are produced from the best available original document. 


\section{RAPID KINETIC FLOW CYTOMETRY}

We have recently described a specialized flow cytometry instrument at the National Flow Cytometry Resource, Los Alamos National Laboratory (Nolan et al., 1995). The Rapid Mix Flow Cytometer (see Figure 1) uses the stepper-motor driven syringes of a Biologic SFM-3 stopped flow mixing device to displace the contents of two sample loops through a mixing tee to a modified Becton Dickinson FACS II flow cytometer. The pitch of the screws of the stepper motors and the sizes of the syringes of the SFM-3 had been fixed by the manufacturer for applications which required mixing and delivery of hundreds of $\mu$ ls of sample in no more than 10's of milliseconds. The volumetric displacement of each sample loop is controlled individually by its own syringe. The software supplied by the manufacturer allowed each syringe and two fast external valves to be controlled in on/off positions.

In order to accommodate rapid delivery of a sample to the flow cytometer, a number of modifications to the instrument had to be made. For optimal flexibility, we replaced the closed syringe and mixing system with syringes with Luer fittings and connected the syringes to manual sample loops and mixing tees. Because the slowest delivery rate available was too high for stable sample flow, the pitch of the screw of the sample delivery syringe was reduced to accommodate the slow delivery rates required by the flow cytometer. In addition, the original software which controlled the syringes and the fast valves had a limit of nine control steps, each with a duration up to 10 seconds. Five of these steps are used to mix and direct sample to the flow cytometer, leaving four steps ( 40 seconds) for measurement. This limitation was exacerbated by the need for additional steps which could control a more complex mixing protocol such as a second mix. We modified the Biologic syringe control software code to allow up to 40 individual control steps, allowing kinetic measurements of over five minutes in duration. The resulting system has sufficient programming flexibility to allow a variety of sample mixing and dilution protocols.

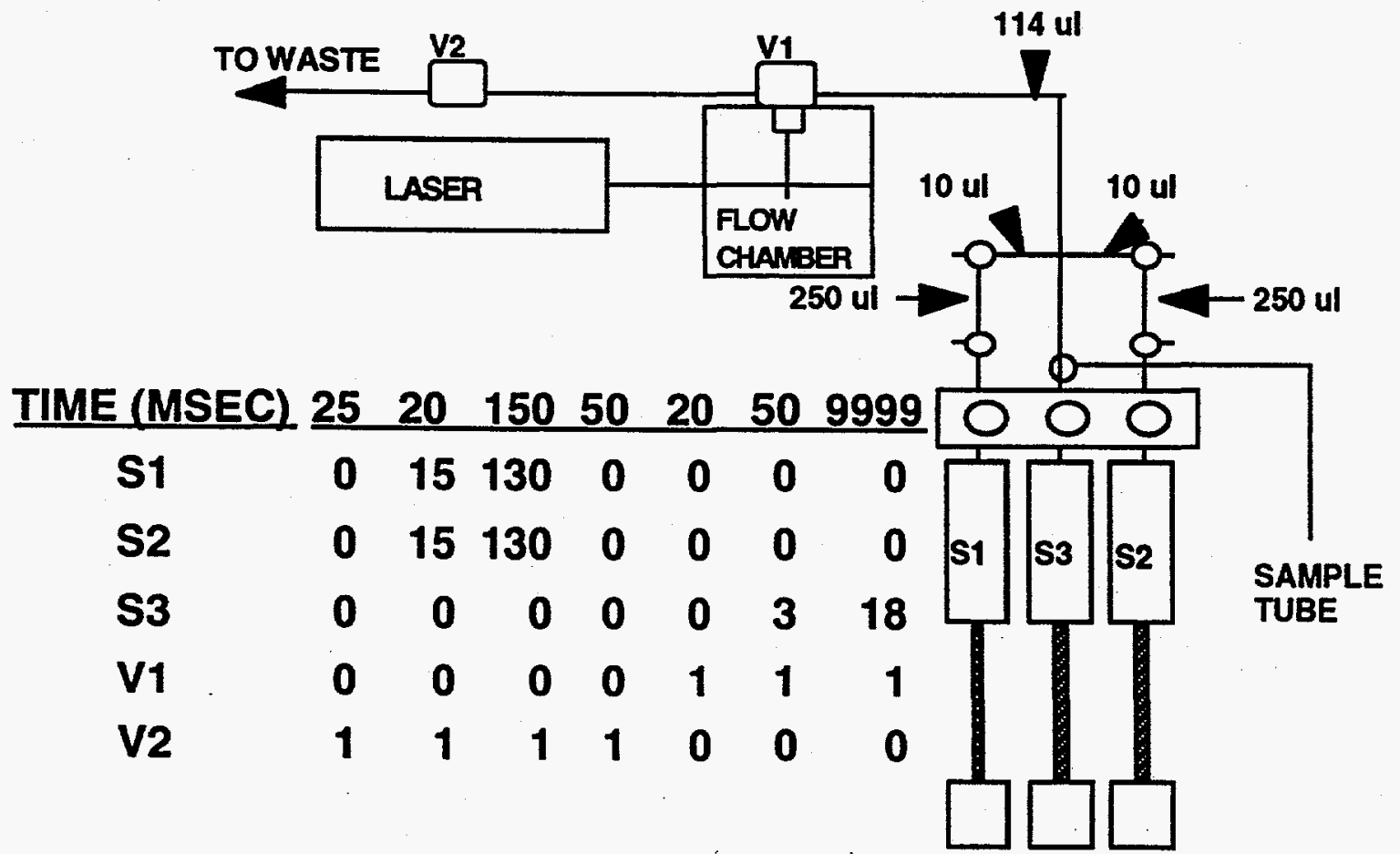

Figure 1. Rapid Mix Flow Cytometer and Mixing Sequence. The mixer is connected to the cytometer by sample loops, mixing " $T$ " or " $\mathrm{X}$ ", and delay lines. The device consists of three syringes ( $\mathrm{S} 1, \mathrm{~S} 2$, and $\mathrm{S} 3$ ) each driven by a stepper motor. Each syringe has a manual valve which allows the syringe to be filled with buffer or the syringe to be connected to the $250 \mu \mathrm{l}$ sample loop. The sample loops are filled manually. The mixing of the sample occurs at the " $X$ " where the lines from the 3 syringes meet. The sample is pushed through the delay line through V1 and V2. A nozzle holder with a $1.5 \mu l$ dead volume holds a 3 way fast switching valve (V1) to control sample flow to the cytometer. V2 controls sample flow to waste. The computer controlled mixing sequence has several phases. In the first phase, the valve position is initialized ( $25 \mathrm{msec})$. Next, the samples in $S 1$ and $S 2$ are pushed in two steps $(20$ and $150 \mathrm{msec}$ ) through the $X$. (The values in the table represent the 
volume delivered in $\mu$ ls during the listed time interval). These two steps advance the contents of the sample loops through the $\mathrm{X}$, then mixes the contents of the sample loops, fills the delay line and expels any old sample beyond V1 and V2. Then, the system is allowed to relax for $50 \mathrm{msec}$ to accommodate pressure changes that result from the mixing. The fast valves VI and $\mathrm{V} 2$ are switched $(20 \mathrm{msec}$ ) so that the sample flow is redirected toward the nozzle. The slow syringe (S3) is used to clear the dead volume between V1 and the nozzle $(50 \mathrm{msec})$. The mixed sample is delivered to the cytometer at a rate of 1.8 ul/sec. The cytometer retains the ability to operate in the traditional, or slow, sample delivery mode, drawing sample from a sample tube when the fast mixer is not being used. A voltage ramp triggered by the mixer computer logs the time base as a cytometer parameter. Two parameter histograms are analyzed and mean fluorescence vs. time is calculated.

Tests validated the performance of the instrument. Fluorescent beads were used to determine the early time detection, uniformity of mixing, and proportioning capability of the syringes. When beads were loaded into one of the sample loops and mixed with an equal volume of buffer, the beads appeared at the laser beam and fluorescence of the bead population was detectable within $300 \mathrm{msec}$. By gating the population on light scatter, it was possible to resolve cells positioned in the laser even though the sample flow was turbulent. Complete mixing of sample in this time frame was demonstrated using potassium iodide to quench the fluorescence of beads surface labeled with FITC. At the earliest times measured; the FITC fluorescence of beads mixed with iodide was quenched compared to samples mixed with buffer. This test confirms that sample mixing is complete by the time particles reach the laser beam. Further tests established the ability of the instrument to quantitatively proportion sample, a necessity for accurate dose-response studies. Cell studies, involving the formyl peptide receptor on neutrophils, were used to define binding and dissociation of fluorescent ligands. A description of the instrument design, operation, and testing has been published (Nolan et al. 1995).

We have conducted physiological experiments to define the instrument capabilities. We examined the calcium response of neutrophils to a series of concentrations of formyl peptide (Fig. 2). We examined the behavior of the cell populations following stimulation with formyl peptide (left panel) To a large extent, cells are found either with resting or elevated calcium levels, in an apparent all-or-none response. The rapid transit of the cells through the device had negligible impact on resting levels of calcium or responses compared to normal cytometric analysis. The right panel shows the time course of the calcium response to several ligand concentrations. At room temperature, there was a delay between the time of the stimulus administration and the cell response which increased as the stimulus concentrations was reduced, but the cell response remained essentially all or none.
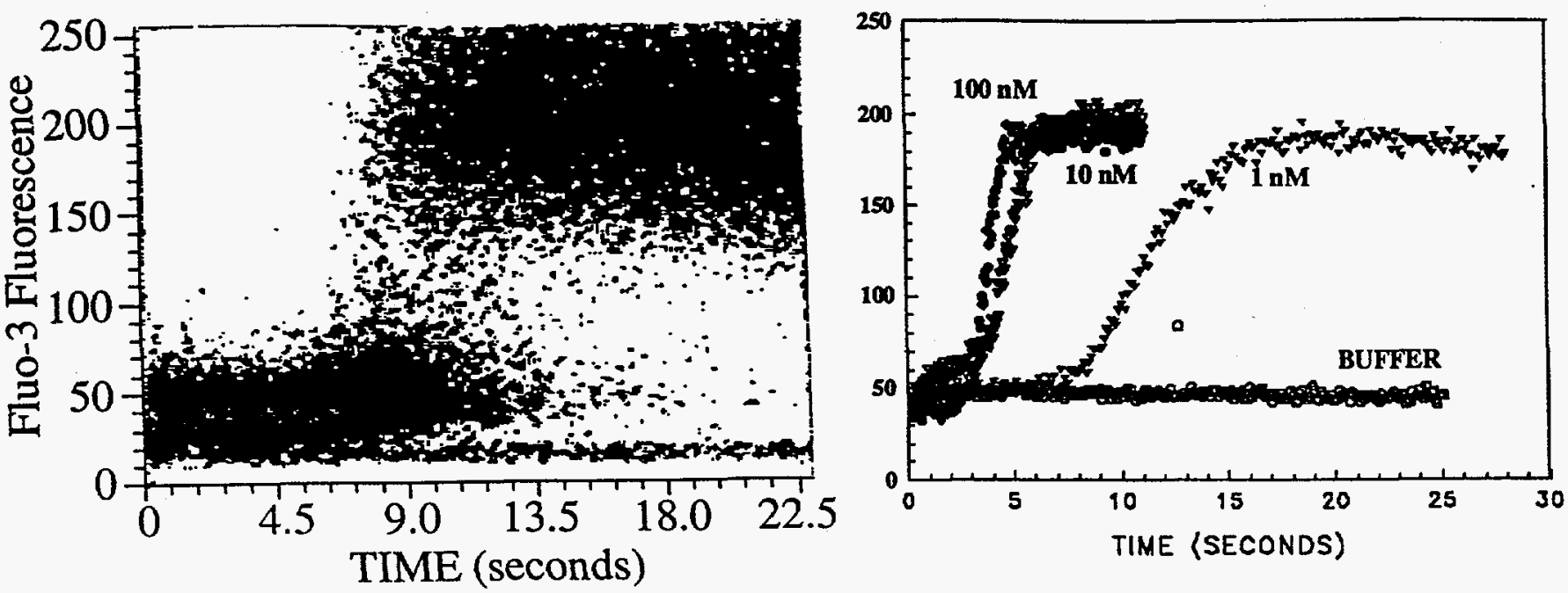

Figure 2. Kinetic Measurements of Formyl Peptide Induced $\mathrm{Ca}^{++}$Fluxes in Neutrophils. The left panel is a dot plot of the intracellular calcium response (Fluo-3 ) vs. time for $1 \mathrm{nM}$ peptide. The right panel shows the calculated mean response for several peptide concentrations. 
We conducted a series of ligand binding experiments to further define the capabilities of the instrument. Fig. 3 shows the kinetics of formyl peptide ligand binding to neutrophils. After the sample mixing, the sample is boosted into the flow cytometer. Associated with the elevated sample flow is a transient turbulence during which the sample stream expands and the ligand detection is perturbed (Fig. 3A). Because the perturbation is detected both in the total binding and non-specific binding kinetics, the specific binding (Fig. 3B) resolves ligand binding even at early times.
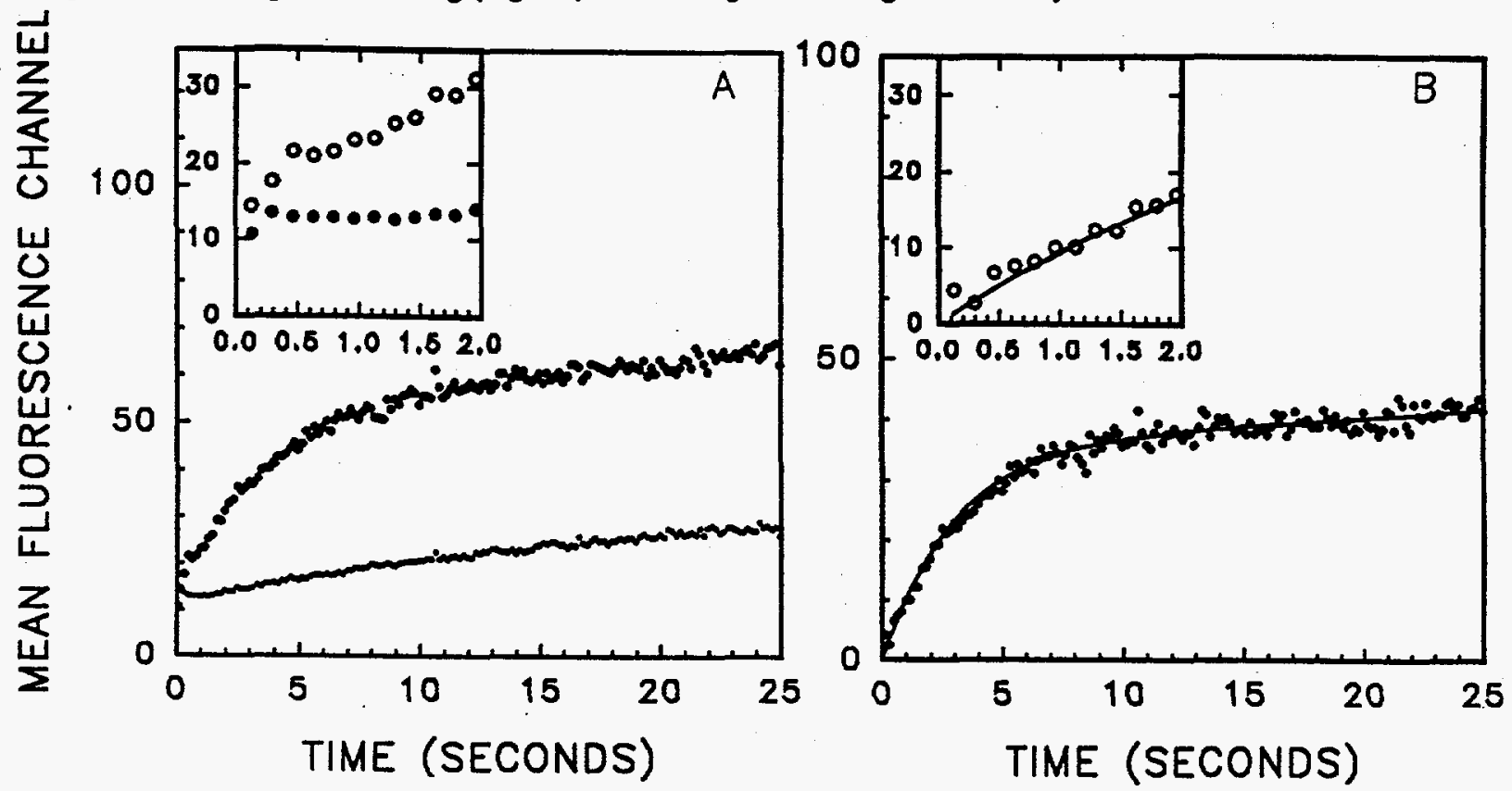

Figure 3. Rapid kinetic measurement of FITC formyl peptide binding to cells. Panel A shows total (broken line) and nonspecific ligand binding kinetics. Panel B shows specific binding kinetics. Insets show early time.

To further evaluate early time performance of the instrument, we examined dissociation of a low affinity ligand (F-met-glyphe-phe-lys-FITC) bound to a mutant receptor (Figure 4). The cells were labeled with high concentrations of ligand, mixed in the rapid mix cytometer to dilute free ligand, then observed for the dissociation of the bound ligand. These experiments indicated that sub-second dissociation processes of low affinity ligands could be detected with the new technology

Figure 4. Rapid Dissociation of a Low Affinity Ligand from a Mutant Formyl Peptide Receptor. The data are expressed as peptide molecules bound per cell. This receptor mutants replaces Aspartate 71 in the second transmembrane domain by Alanine and retains the receptors in a state of low binding affinity. Data compare the rapid dissociation of the low affinity ligand (open symbols) with a slowly dissociating control ligand (closed symbols) following dilution from $100 \mathrm{nM}$ to $10 \mathrm{nM}$.

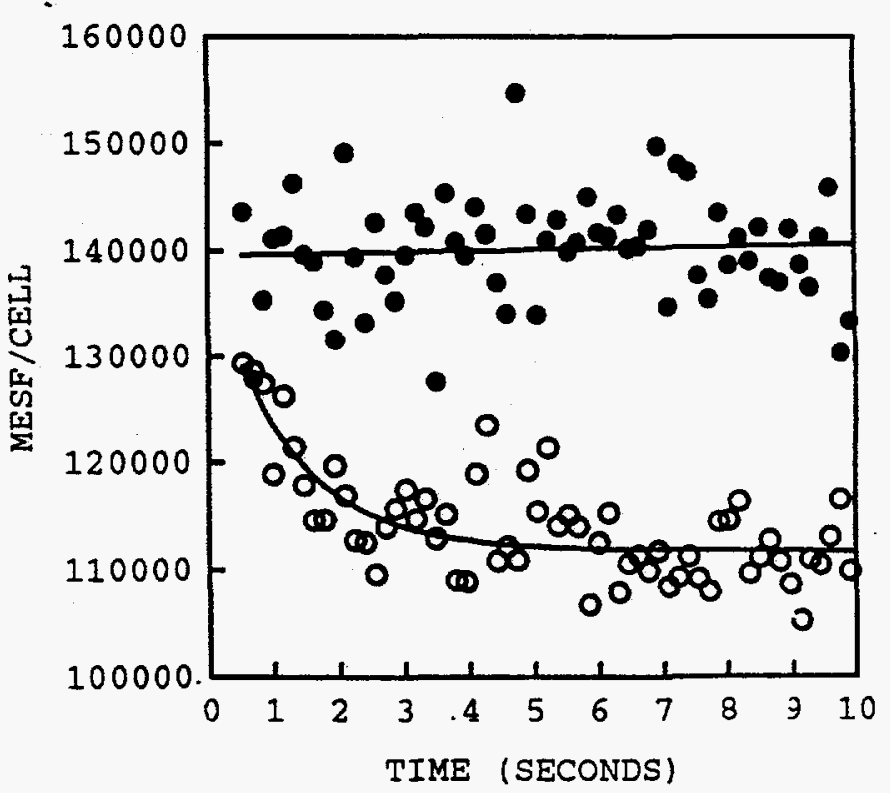


Two-Step Mixing. Two-step mixing experiments involve mixing cells with a ligand which stimulates the cells. Then the stimulus is inhibited by adding an antagonist to the same receptor. This allows a pulse of stimulation in which cell responses such as intracellular calcium or ligand binding and dissociation can be evaluated over time. We modified the rapid mix cytometer for double mix protocols by splitting the sample line from $\mathrm{SI}$ and adding a second mixing tee. In these experiments, S1 was used to mix cells and fluorescent ligand in the first step, S2 was used to stop the binding of the ligand by adding non-fluorescent ligand, then S3 was used in the normal manner for sample boost and delivery. A schematic of this protocol is shown in Fig. 5 and the results for formyl peptide binding and dissociation on a transfected cell line are shown in Fig. 6. We found that we were readily able to program multiple mixing steps within time frames shorter than 10 seconds. In principle, these steps can be compressed for sub-second processes.

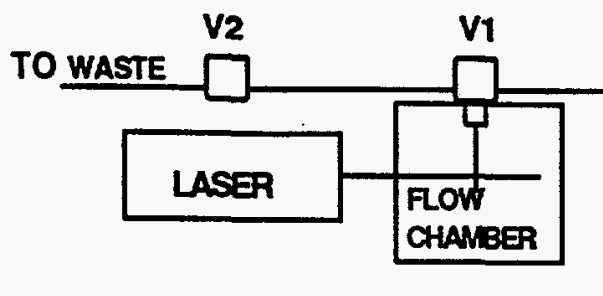

TIME (MSEC $25 \quad 550$ WAIT $600 \quad 50 \quad 20 \quad 50 \quad 9999$

$\begin{array}{lcccccccc}\text { S1 } & 0 & 550 & 0 & 300 & 0 & 0 & 0 & 0 \\ \text { S2 } & 0 & 0 & 0 & 300 & 0 & 0 & 0 & 0 \\ \text { S3 } & 0 & 0 & 0 & 0 & 0 & 0 & 3 & 18 \\ \text { V1 } & 0 & 0 & 0 & 0 & 0 & 1 & 1 & 1 \\ \text { v2 } & 1 & 1 & 1 & 1 & 1 & 0 & 0 & 0\end{array}$

500 ul

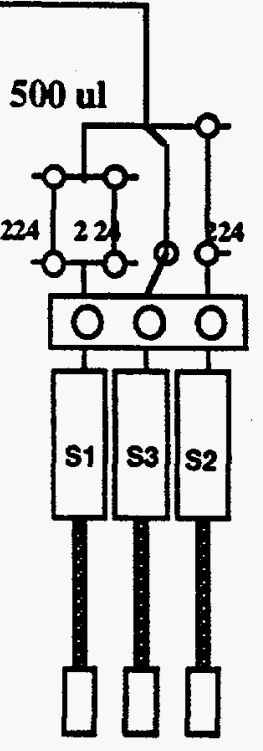

Figure 5. Rapid Mix Flow Cytometer Configuration and Sequence for Double Mix Experiments. We reconfigured the sample and delay lines to enable one syringe to mix equal volumes from two sample loops, allowing the other syringe to displace the contents of a third sample loop in a second mixing step. This configuration is depicted schematically along with the control sequence for the Biologic syringes and the fast-acting solenoid valves. First, valve 2 (V2) is opened, so that all fluid is displaced to waste (Step 1). Syringe 1 (S1) is then activated to displace the contents of two sample loops through a mixing tee into the first delay line (D1) (Step 2). In this step, buffer from S1 is partitioned equally resulting in the mixing of equal volumes of reagents from these two sample loops. The mixed sample is allowed to age in D1 for the desired amount of time (Step 3), before S2 and S1 are activated to mix the contents of sample loop 2 with the contents of D1 through a second mixing tee into delay line 2 (D2) (Step 4). Once this second mixing step occurs, the remainder of the mixing protocol is the same as for a single step mix. There is a short $(20-50 \mathrm{msec})$ waiting period to allow any turbulence from the second rapid mix to subside (Step 5), then V2 is closed and valve 1 (V1) is opened (Step 6) so that all fluid is displaced to the cytometer. The signal to open Vl also starts a voltage ramp, which provides the time base. The dead volume of the cytometer inlet line is rapidly displaced by a boost from S3 (50 msec, Step 7). The contents of D2 is delivered at a slow rate (1.8 ul/sec) to the point of measurement (Step 8), and the fluorescence of the sample is measured.

Applications of Two-Step Mixing Experiments. In many cellular processes, the binding interactions between soluble ligands and cell surface molecules receptors change over time. Because the time frame of cell responses may be in seconds, the interactions between ligands and receptors and subsequent receptor signaling steps may occur in the sub second time frame. Mechanistic studies therefore require measurements of the time dependent changes on the appropriate time scale. One example of such experiments involve the addition of ligand to cells to allow binding to occur for a short period of time, followed by addition of unlabeled ligand or antagonist to induce dissociation (Fig. 6). The figure compares two types of experiments: a binding experiment and a dissociation experiment. In the first experiment, cells and fluorescent ligand are 
contained in the two sample loops which are controlled by $\mathrm{S} 1$ and the association time course is measured. In the dissociation experiment, at intervals after the first mix, the operation of S1 and S2 mixes unlabeled ligand with the labeled cells to induce dissociation. The interval between the first and the second mix can be controlled with great precision.

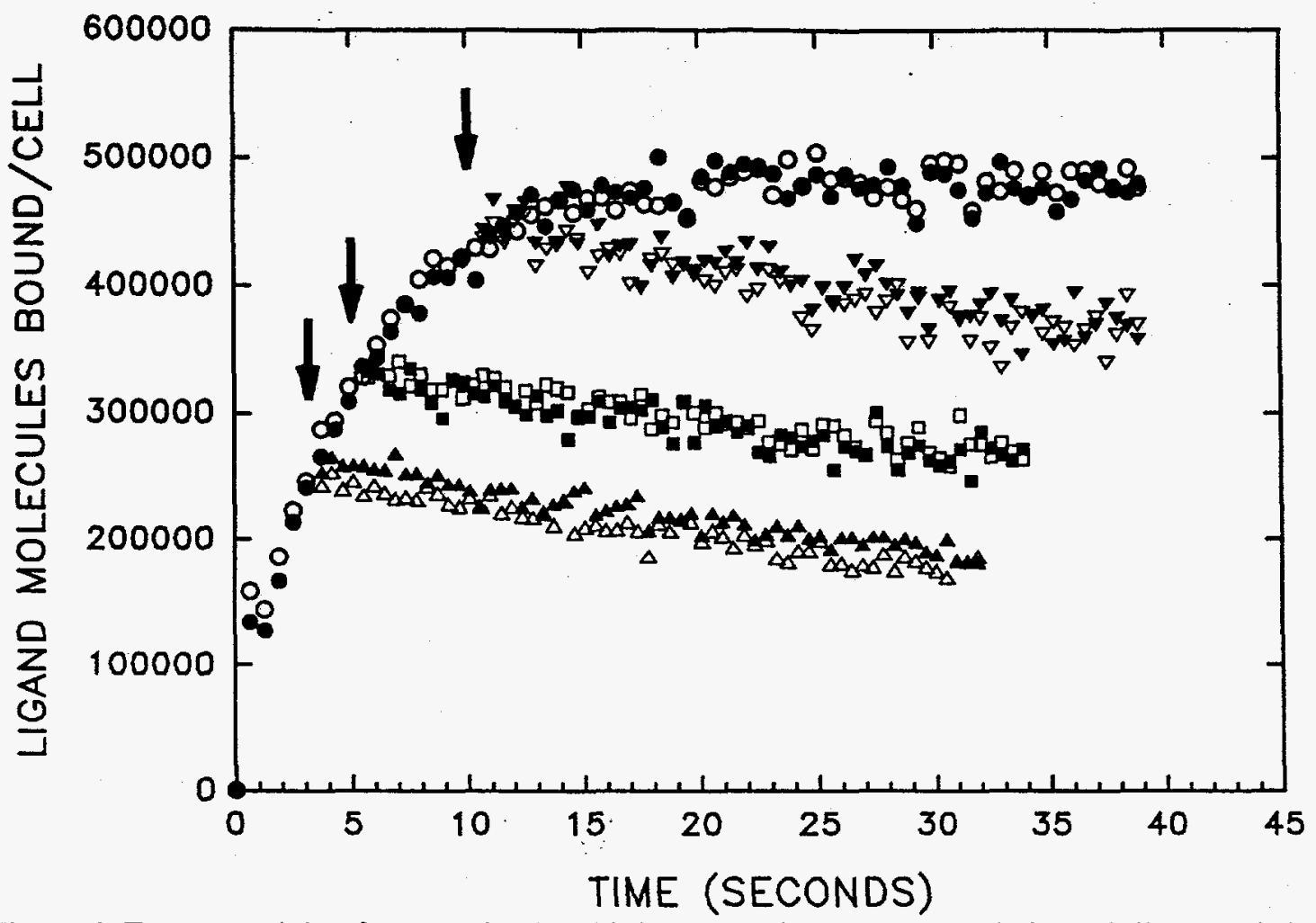

Figure 6. Two-step mixing for analysis of rapid time-dependent receptor evolution. Cells and labeled ligand were loaded into the two sample loops controlled by S1, and buffer plus unlabeled ligand was loaded into the sample loop controlled by S2. S1 mixed the contents of the sample loops in equal proportions. The mixed sample was then directed to the sheath stream to measure the association kinetics, or allowed to age in the delay line. After binding had proceeded for 3, 5 , or 10 seconds, the contents of delay line 1 were mixed with the contents of sample loop 2 to fill delay line 2 , and the twice mixed samples were delivered to the flow cytometer for measurement of dissociation of labeled ligand.

Another application under development is intended to extend the range of sensitivity of flow cytometry for detecting ligand binding. Our experience has shown that once ligand concentrations exceed $\sim 100 \mu \mathrm{M}$, it becomes difficult to evaluate ligand which is specifically associated with cellular receptors in conventional flow cytometry. While it is possible to combine a manual washing step between the time that cells are labeled and the time they are analyzed in the flow cytometer, ligands with dissociation times shorter than the wash steps would be lost from the cell. With a rapid mix or multi mix capability, cells could be labeled with high concentrations of ligand, then a rapid dilution step could allow dilution of free ligand away from the cells and observation of the cells prior to the time in which bound ligand had dissociated from the receptors.

\section{CONTROL OF TURBULENCE AND AUTOMATION}

The system described above uses three syringes for controlling sample mixing and delivery but none of the syringes are available to control the sheath flow. We have investigated the possibility of using a syringe to control sheath flow so that total flow through the flow cell could remain constant during sample delivery. A schematic diagram of the Alitea FIA system is shown in Figure 7. The flow-injection hardware supplied by the manufacturer was supplemented with additional syringe pumps and valves. The control software was also modified by the manufacturer to shorten the command cycle times. The unit consists of a control module with one internal valve, one external valve, three added extenal syringe pumps and programmable Windows ${ }^{\mathrm{mm}}$ based control software. Each of the syringes may be replaced with barrels ranging from $100 \mu \mathrm{ls}$ to $10 \mathrm{mls}$. At the present time, separate computers are used for FIA control and data acquisition for the flow cytometer. 


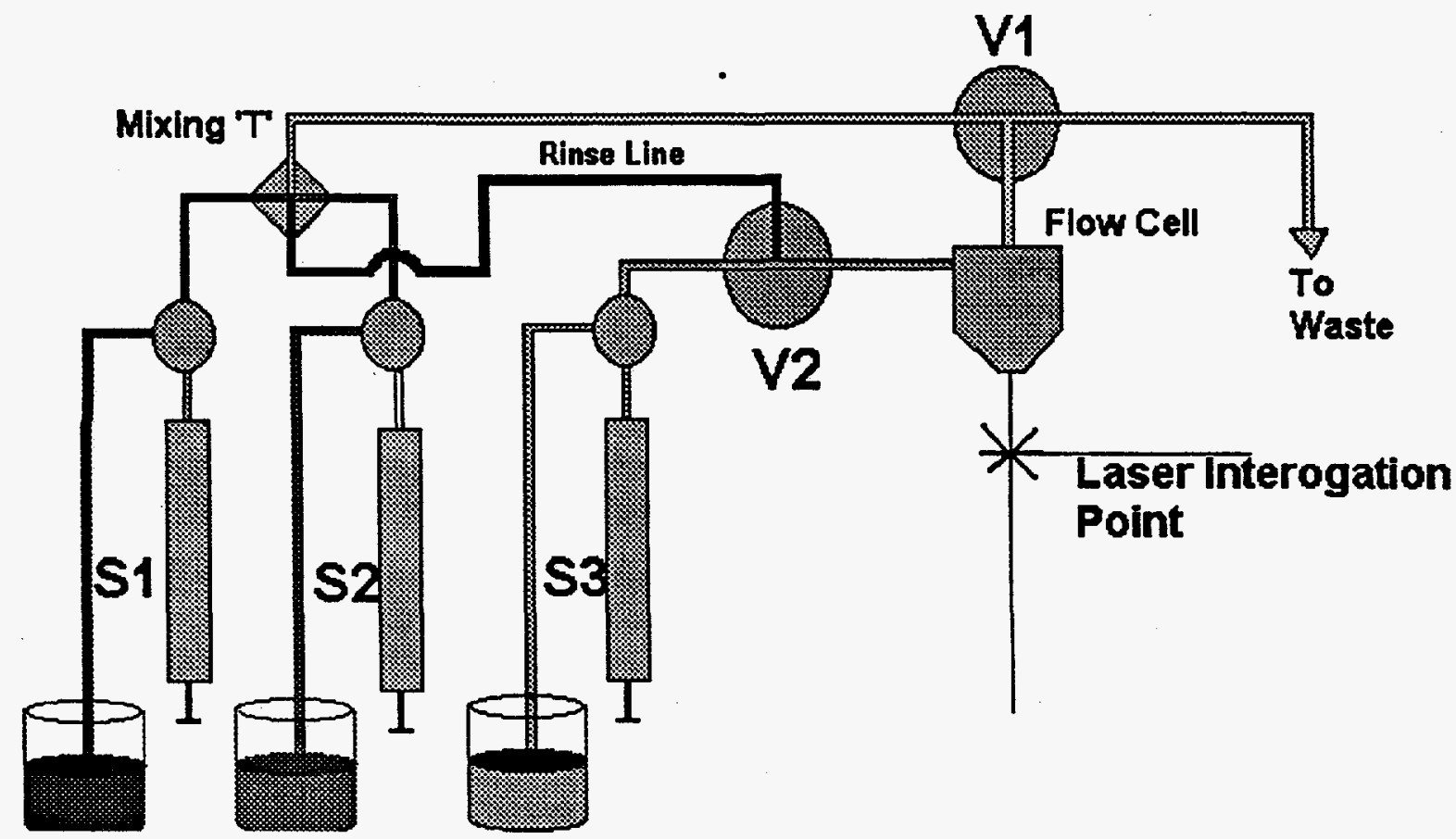

Figure 7. Schematic of the FIA Flow Cytometer using the Alitea Control System. S1 is a $0.5 \mathrm{ml}$ syringe pump used to dispense sample (either cells or beads) to the mix line. S2 is a $1 \mathrm{ml}$ syringe pump used to deliver reactant to the mix line and then deliver the sample-reactant mixture to the flow cytometer. $\mathrm{S} 3$ is a $5 \mathrm{ml}$ syringe pump used to dispense sheath buffer. VI is a Valco 6-position valve, positioned as close to the flow cell sample-input port as possible, $\sim 3 \mathrm{~cm}$. V2 is an identical valve used to direct the sheath buffer to the flow cell, during normal operation, redirecting sheath to the mixing line, for rinsing between samples.

TABLE 1. Sample Delivery Control Sequence

\begin{tabular}{|c|c|c|c|c|c|}
\hline TIMEImsec & $\begin{array}{c}\text { S1(sample) } \\
\mu \mathrm{V} / \mathrm{sec}\end{array}$ & $\begin{array}{c}\text { S2(reagent) } \\
\mu \mathrm{l} / \mathrm{sec}\end{array}$ & $\begin{array}{c}\text { S3(sheath) } \\
\mu \mathrm{l} / \mathrm{sec}\end{array}$ & $\begin{array}{c}\text { V1 } \\
\text { position }\end{array}$ & Time Pulse \\
\hline$-8,000$ & 0 & 0 & 8 & Waste & Off \\
\hline-100 & 0 & 0 & s. & Waste & Off \\
\hline-50 & 0 & x & 2 & Waste & Off \\
\hline 0 & 0 & 75 & 2 & Waste & 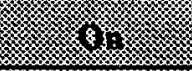 \\
\hline+25 & 0 & 75 & 2 & Waste & (3) \\
\hline+75 & 8 & 75 & 2 & Waste & Off \\
\hline+375 & 75 & 75 & 2 & 1) & Off \\
\hline+425 & 2 & 75 & 2 & Flow Cell & Off \\
\hline+475 & 2 & 75 & 8 & Flow Cell & Off \\
\hline+525 & 2 & $(3)$ & 48 & Flow Cell & Off \\
\hline
\end{tabular}


A sequence of commands has been developed, Table 1, which allows the sheath flow to be reduced during sample introduction. The values in the table represent the flow in $\mu \mathrm{l} / \mathrm{sec}$ during the listed time interval. The command sequence requires that each device be controlled by a separate command, so each device is individually given a start and end time and description of its activity as highlighted in the shaded boxes. For the syringes, the activity is the flow rate per second. For the multi port valves, a direction must be specified. In our sequence, stable sheath flow is established 8 seconds prior to sample mixing. The next five commands follow quickly in succession. First, sheath flow is reduced to $2 \mu \mathrm{ls} / \mathrm{sec}$. Second, the syringe controlling the soluble reactant is activated. The third and fourth commands create a time-stamp in the data file which is discussed elsewhere (Seamer et al., submitted). In the fifth command sample and reactant mixing occurs, as cell sample flow is begun. The total time from creation of the time-stamp to the onset of sample mixing is 75 msecs, and depends in part upon the Alitea software which checks that each device is performing its defined function. Sample and reactant mixing continues at $150 \mathrm{ul} / \mathrm{sec}$ total flow rate for 300 msecs. Because the delay line between the mixing tee and the V1 valve holds 45 $\mu$, enough volume has been mixed, so that freshly mixed sample has been pushed beyond V1. At that time, V1 changes position, directing the mixed sample towards the flow cell. 50 msecs later, the rate of sample delivery is slowed to $2 \mu 1 / \mathrm{sec}$, the stable analyses rate. The rate of sheath flow is increased to $48 \mu \mathrm{l} / \mathrm{sec}$ and stable flow is re-established.

The performance of this system has been characterized in several ways including restoration of stable flow, accuracy and reproducibility of mixing, and automation of sampling by software control. Figure 8 demonstrates that the instrument was achieving thorough mixing over a range of sample delivery volumes prior to the cells reaching the laser interrogation point. To do this we took advantage of the property of iodide to quench the fluorescence of fluorescein labeled beads. The left panel shows a dot-plot of fluorescence versus time when $125 \mathrm{mM} \mathrm{KI}$ is added to beads (upper) and the calculated mean fluorescence intensity after mixing (lower). Together, these two plots demonstrate that within $200 \mathrm{msec}$ after the beginning of sample delivery (in this case at about $600 \mathrm{msecs}$ ), the fluorescence intensity is already stable as a result of complete mixing and control of turbulence. If the sheath were not controlled, and left at a constant flow rate during sample injection, recovery of stable flow of the sample would require $\sim 1 \mathrm{sec}$. As shown in the right panel, the quenching experiment can be controiled from the terminal by varying the proportion of the volumes delivery from S2 and S3. The result is a Stern-Vollmer plot of quenching as a function of the KI concentration. Between samples the valve positions are reconfigured so that $\mathrm{Sl}$, the sheath syringe, delivers buffer to rinse the sample lines, effectively automating the delivery process. A sampling cycle of 10-20 sec could be achieved by cycling the control sequence.
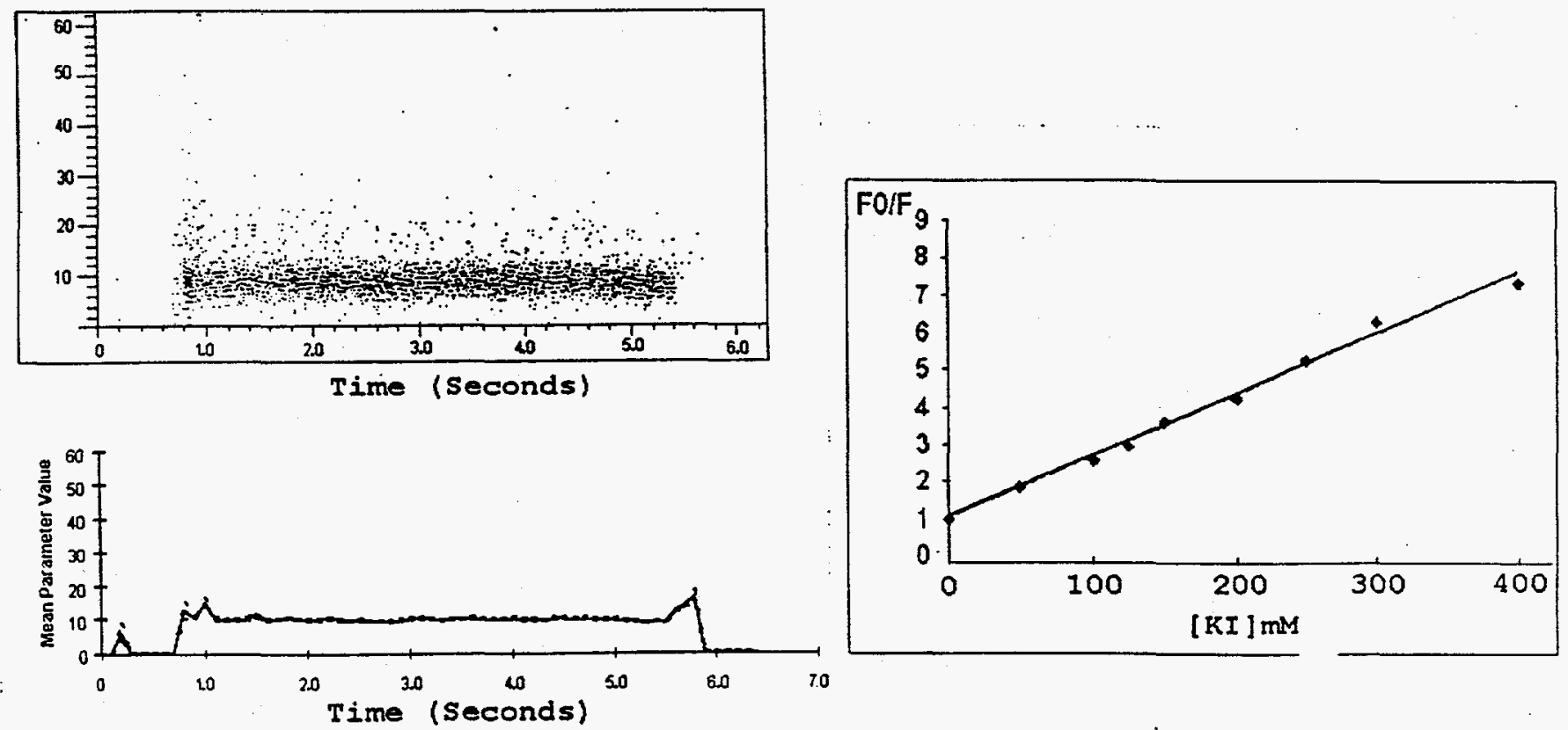

Figure 8. Quenching of fluorescence during controlled sheath delivery. Left. Dot plot and mean channel value of fluorescence after mixing with Potassium iodide during controlled sheath dzlivery. Note that the sample is quenched by the time stable delivery is established Right. Stern-Volmer plot of quenched fluorescence vs. KI concentration. The data were obtained in a semi-automated fashion by regulating the mixing proportions of S1 and $\mathrm{S} 2$. 


\section{CONCLUSIONS}

Flow cytometry offers sensitivity for a variety of bead or cell based assays of molecular assembly and cell response. These are likely to find basic, clinical, and commercial applications. We have investigated several types of robust sample handling systems. The first system, at a cost of $\sim \$ \$ 0,000$ was intended to provide optimal time resolution, but was limited by the number of its syringes to sample mixing and delivery. Turbulent flow characteristics had to be resolved optically. However, multiple step mixing protocols could be performed. The second system, at a cost of $\sim 10,000$ offered flexibility, having the ability to control multiple valves, and syringes of variable barrel size and delivery rate. The system could be used to control both sheath and sample, allowing stable sample flow within 200 msecs of sample mixing. Both systems allow the potential of programmable, terminal control sample delivery. We are developing new systems which combine the best features of both.

\section{ACKNOWLEDGMENTS}

This work was supported by NIH RR01315, HHMI, DOE NN20 and the Cancer Center of the University of New Mexico.

\section{REFERENCES}

Dive C, Workman P, Watson JV, "Improved methodology for intracellular enzyme reaction and inhibition kinetics by flow cytometry". Cytometry 8, pp. 552-61, 1987.

Fay SP, Posner RG, Swann WN, and Sklar LA, "Real-time analysis of the assembly of ligand, receptor, and G protein by quantitative fluorescence flow cytometry". Biochemistry 30, pp.5066-5075, 1991

Kachel V, Glossner E, and Schneider H; "A new flow cytometric transducer for fast sample throughput and time resolved kinetic studies of biological cells and other particles". Cytometry 3, pp. 202-212, 1982.

Kelley KA, "Sample station modification providing on line reagent addition and reduced sample transit time for flow cytometers". Cytometry 10, pp. 796-800, 1989.

Lindberg W, Scampavia LD, Ruzicka J, and Christian GD, "Fast kinetic measurements and on-line dilution by flow injection cytometry". Cytometry 16, pp. 324-330, 1994.

Martin JC and Schwartzenruber, DE, "Time: A new parameter for kinetic measurements in flow cytometry". Sçience 207, pp. 199-201, 1980.

Nolan JP, Posner RG, Martin JC, Habbersett RC, and Sklar LA, "A rapid mix flow cytometer with subsecond kinetic resolution". Cytometry 21, pp. 223-229, 1995.

Nolan JP, Chambers, JD, Sklar LA, "Cytometric Approaches to the study of receptors". In, Cytometric Cellular Analysis, Vol. 1. Phagocyte Function: A guide for Research and Clinical Evaluation (JP Robinson and G Babcock, eds). J. Wiley \& Sons. (In press, 1998)

Omann GM, Coppersmith W, Finney DA, and Sklar LA, "A convenient on-line device for reagent addition, sample mixing, and temperature control of cell suspensions in flow cytometry". Cytometry 6, pp. 69-73, 1985.

Scampavia LD, Blankenstein G, Ruzicka J and Christian GD. "A coaxial jet mixer for rapid kinetic analysis in flow injection cytometry". Analytical Chemistry 67, pp. 2743-2749, 1995.

Seamer LC, Kuckuck F and Sklar LA, "Sheath control to defeat turbulence in rapid mix flow cytometry". Cytometry, submitted

Sklar LA, “ Real-time spectroscopic analysis of ligand-receptor dynamics”. Ann. Rev. Biophys. Biophys. Chem. 16, pp. 479$506,1987$. 